\title{
Antihistamines in the treatment of pruritus in psoriasis
}

\author{
Anna Domagała ${ }^{1}$, Jacek Szepietowski², Adam Reich ${ }^{2,3}$ \\ ${ }^{1}$ Dermatology Department, Regional Specialist Hospital, Research and Development Centre, Wroclaw, Poland \\ ${ }^{2}$ Department of Dermatology, Venereology and Allergology, Wroclaw Medical University, Wroclaw, Poland \\ ${ }^{3}$ Department of Dermatology, University of Rzeszow, Rzeszow, Poland
}

\author{
Adv Dermatol Allergol 2017; XXXIV (5): 457-463
}

DOI: https://doi.org/10.5114/ada.2017.71112

\begin{abstract}
Aim: To evaluate the efficacy of antihistamines in reducing pruritus in psoriasis, 61 patients were randomized to be treated for 1 week with clemastine $(n=20)$, levocetirizine $(n=21)$ or placebo $(n=20)$.

Material and methods: All patients received the same routine antipsoriatic treatment. Itch intensity was assessed with VAS and the Itch Questionnaire, and hand movements during sleep were counted with an accelerometer. Results: There was a statistically significant decrease in mean VAS scoring in clemastine and levocetirizine groups $(p<0.001)$, but not in the placebo group. Questionnaire scoring decreased significantly during the study in all study groups, with the greatest improvement noted in the clemastine group. The number of wrist movements during sleep did not differ significantly between groups.

Conclusions: Antihistamines of the first and second generations seem to be effective in reducing itch in patients with psoriasis, albeit the antipruritic effect is rather moderate. These observations need to be confirmed on larger patient groups.
\end{abstract}

Key words: pruritus, psoriasis, antihistamines.

\section{Introduction}

Psoriasis is one of the most common chronic inflammatory skin diseases, which affects approximately 1-2\% of the world general population. The etiopathogenesis of psoriasis has not been yet fully understood, but genetic predisposition, hyperproliferation of keratinocytes, vascular alterations in the skin, upregulation of cytokines, immunological disturbances as well as environmental factors are thought to play an important role in its development $[1,2]$. Several studies have clearly shown that pruritus affects about $70 \%$ to $90 \%$ of patients with plaque type psoriasis and is often described as the most burdensome symptom of the disease [2-7]. Although the mean severity of itch seems to be lower than in other highly pruritic skin conditions, such as atopic dermatitis or lichen planus, it has been documented that majority of patients with psoriasis consider pruritus to have a negative effect on their quality of life (QoL) [3, 8]. Importantly, there is still no effective anti-pruritic treatment proven for psoriasis as the pathogenesis of itch in this disease has not been fully elucidated. Although histamine has been believed not to play a crucial role in the pathogenesis of psoriatic pruritus, antihistamines (especially the first-generation ones with additional sedative properties) are often used in daily clinical practice to reduce psoriatic itch. It has been suggested that a potential anti-pruritic effect, if any, is related to the sedative effect of the firstgeneration antihistamines and that selective histamine blockade does not improve itch, but this effect has not been studied well so far.

\section{Aim}

Therefore, the current investigator-initiated study has been designed as a double-blinded, randomized and placebo-controlled trial to evaluate the potential antipruritic effectiveness of antihistamines of both, the first and second generations, in the treatment of itch in psoriasis patients.

\section{Material and methods}

Patient characteristics

The study was approved by the Ethics Committee of Wroclaw Medical University (agreement No. 485/2012). All patients gave their informed consent

Address for correspondence: Prof. Adam Reich MD, PhD, Department of Dermatology, University of Rzeszow, 2 Szopena St, $35-055$ Rzeszów, Poland, phone: +48 605076 722, e-mail: adi_medicalis@go2.pl

Received: 21.06.2016, accepted: 11.07.2016. 
Table 1. Clinical characteristics of study patients with psoriasis

\begin{tabular}{|c|c|c|c|c|}
\hline Parameter & Clemastine group & Levocetirizine group & Placebo group & $P$-value \\
\hline Number of patients & 20 & 21 & 20 & - \\
\hline Age [years]: & & & & 0.97 \\
\hline Mean \pm SD & $55.1 \pm 14.2$ & $53.9 \pm 13.9$ & $54.2 \pm 17.3$ & \\
\hline (Range) & $(19-76)$ & $(32-80)$ & $(25-86)$ & \\
\hline Gender, n (\%): & & & & 0.8 \\
\hline Female & $13(65.0)$ & $13(62.0)$ & $11(55.0)$ & \\
\hline Male & $7(35.0)$ & $8(38.0)$ & $9(45.0)$ & \\
\hline Duration of psoriasis [years]: & & & & 0.87 \\
\hline Mean \pm SD & $20.2 \pm 16.4$ & $21.4 \pm 14.8$ & $22.8 \pm 16.4$ & \\
\hline (Range) & $(1-58)$ & $(0.3-46)$ & $(1-49)$ & \\
\hline $\begin{array}{l}\text { Number of patients with psoriatic } \\
\text { arthritis, } n(\%)\end{array}$ & $2(10.0)$ & $2(9.5)$ & $1(5.0)$ & 0.82 \\
\hline $\begin{array}{l}\text { Positive family history of psoriasis, } \\
n(\%)\end{array}$ & $9(45.0)$ & $6(28.6)$ & $9(45.0)$ & 0.46 \\
\hline BMI $\left[\mathrm{kg} / \mathrm{m}^{2}\right]$ : & & & & 0.62 \\
\hline Mean \pm SD & $27.3 \pm 5.3$ & $28.8 \pm 5.5$ & $27.6 \pm 4.8$ & \\
\hline (Range) & $(18.4-37.4)$ & $(19.5-39.8)$ & $(20.1-36.5)$ & \\
\hline PASI (Baseline): & & & & 0.44 \\
\hline Mean \pm SD & $12.5 \pm 5.3$ & $13.1 \pm 6.6$ & $14.9 \pm 5.5$ & \\
\hline (Range) & $(6.4-27.6)$ & $(4.6-28.8)$ & $(6.0-28.4)$ & \\
\hline PASI (EOS): & & & & 0.42 \\
\hline Mean \pm SD & $7.1 \pm 4.6$ & $7.0 \pm 4.5$ & $8.6 \pm 3.4$ & \\
\hline (Range) & $(2.7-21.0)$ & $(2.3-16.5)$ & $(3.2-14.8)$ & \\
\hline BSA (Baseline) (\%): & & & & 0.45 \\
\hline Mean \pm SD & $13.6 \pm 6.2$ & $14.5 \pm 9.6$ & $17.1 \pm 10.2$ & \\
\hline $\begin{array}{l}\text { (Range) } \\
\text { (R) }\end{array}$ & $(6-27)$ & $(6-45)$ & $(5-42)$ & \\
\hline BSA (EoS): & & & & 0.45 \\
\hline Mean \pm SD & $13.4 \pm 6.3$ & $14.1 \pm 9.5$ & $16.8 \pm 9.6$ & \\
\hline (Range) & $(6-27)$ & $(6-43)$ & $(5-42)$ & \\
\hline
\end{tabular}

BMI - body mass index, EOS - end of study, SD - standard deviation.

prior to inclusion in the study. A total of 61 adult patients with plaque type psoriasis and concomitant pruritus hospitalized in the Department of Dermatology of the Regional Specialist Hospital in Wroclaw were enrolled in the study. The study group consisted of 24 males (39.3\%) and 37 females (60.7\%) aged from 19 to 86 years (mean age: $54.4 \pm 15.0$ years). All patients were randomly divided into one of the following three groups: group 1 - patients were given clemastine twice daily - one tablet orally $(2 \times 1 \mathrm{mg})$ for 6 days; group 2 - patients were given levocetirizine once daily - one tablet in the evening $(1 \times 5 \mathrm{mg})$ and one placebo tablet in the morning for 6 days; group 3 (control group) - patients were given placebo twice daily - one tablet for 6 days (to ensure blindness of the study all tablets were put in the starch petal by the hospital pharmacist and were given to the patients by a physician not involved in the study assessments). Twenty (32.8\%) patients were randomly assigned into group 1, 21 (34.4\%) patients into group 2 and 20 (32.8\%) patients into group 3. Detailed characteristics of the enrolled patients are shown in Table 1.

\section{Inclusion and exclusion criteria}

Adult patients (aged 18 years old or older) with plaque type psoriasis and concomitant pruritus were enrolled into the study. The diagnosis of plaque type psoriasis had to be made at least 3 months prior to the enrollment. Patients with a non-plaque form of psoriasis (e.g. erythrodermic, guttate or pustular psoriasis) 
were excluded. Any potential patient who met any of the following criteria was also excluded from the study: NYHA III or NYHA IV heart failure, itch that could be induced by other dermatological or systemic diseases, receiving any medications which are known to cause pruritus or have an antipruritic effect. Women who were pregnant or breast-feeding as well as patients with contraindications to antihistamines were also excluded. Patients who were enrolled into the study were not allowed to receive any antihistamines, phototherapy or systemic treatment of psoriasis within 2 weeks prior to randomization.

\section{Study design}

The study was designed as a double-blinded, randomized and placebo-controlled trial. All patients underwent a careful anamnesis and physical examination in order to collect demographic and clinical data. Patients were examined on the first day of admission before any anti-psoriatic and antipruritic treatment was initiated. All patients received the same routine treatment of psoriatic skin lesions (topical treatment with keratolytics followed by anthralin and UVB $311 \mathrm{~nm}$ phototherapy). The whole study period was 7 days for each patient. Such a short period was chosen for two reasons: all patients received antipsoriatic treatment as in-patients and many of them already demonstrated a significant improvement of skin lesions after 1 week's stay at the hospital and subsequently, marked pruritus reduction. Secondly, because of administrative reasons some patients could not stay longer in the hospital and in order not to have a high drop-off rate, the study period had to be kept short. At baseline (day 1) and at the end of the study (day 7), the severity of psoriasis with Psoriasis Area and Severity Index (PASI) and body surface area (BSA) [9-12] and quality of life according to Dermatology Life Quality Index (DLQI) $[13,14]$ were assessed. Patients were asked to

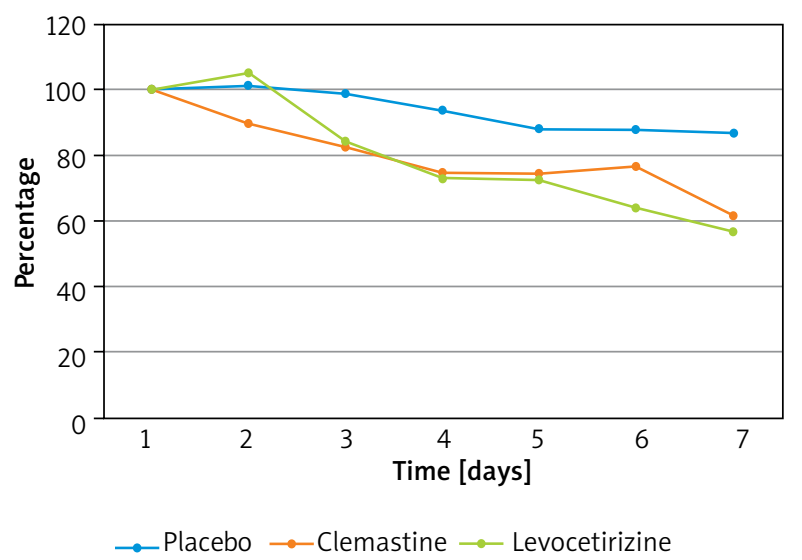

Figure 1. Relative change of the visual analog scoring in the treated groups assess pruritus intensity over the last $24 \mathrm{~h}$ according to the 10-point visual analogue scale (VAS) [15, 16], and the Itch Questionnaire which was used successfully in our previous studies on pruritus [17]. Within next days of the study (day 2 through day 6) patients only assessed itch intensity using VAS diary.

In addition, an accelerometer ActiSleepPlus (ActiGraph, FL, USA, 850.332.7900) was worn on the wrist of the dominant hand of the subject for 6 consecutive nights to measure the hand movements during sleep. This method, called actigraphy, was previously used to assess pruritus in different skin disorders $[18,19]$. The digital accelerometer output was exported to software for analysis on day 7 .

\section{Statistical analysis}

All data were analyzed statistically with Statistica 10.0 (Statsoft, Krakow, Poland). Means, standard deviations (SD), median values and frequencies were calculated. The differences between the groups of patients were analyzed using the Student's t test for independent variables, Mann-Whitney $U$ test, analysis of variance (ANOVA) and multiple regression analysis, where appropriate. Correlations between analyzed parameters were verified by using Spearman's rank correlation test ( $\rho-$ correlation coefficient). $\chi^{2}$ test was used to determine whether there was a significant difference between the expected and observed frequencies in one or more categories. The results were considered statistically significant, if $p$-value was less than 0.05 .

\section{Results}

The baseline PASI was similar in all study subgroups (clemastine: $12.5 \pm 5.3$ points, levocetirizine: $13.1 \pm 6.6$ points and placebo: $14.9 \pm 5.5$ points, $p=0.44$ ) and reduced significantly in all patients: PASI for the clemastine

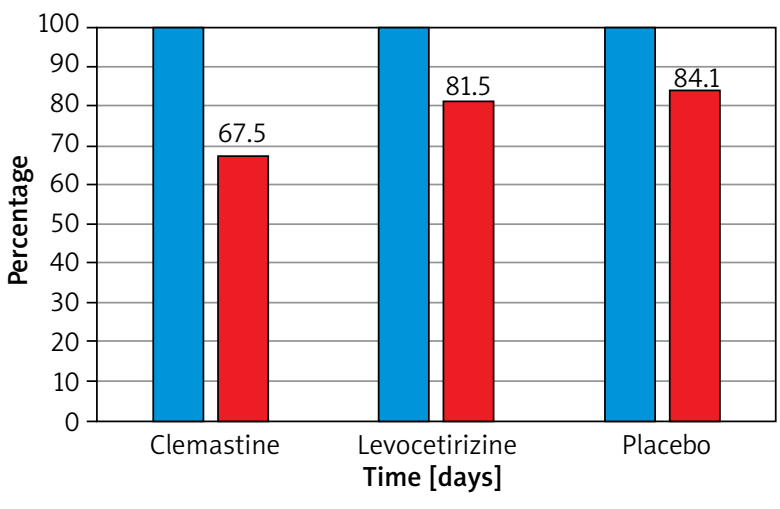

$\square$ Itch Questionnaire Day $1 \square$ Itch Questionnaire Day 7

Figure 2. Relative change of Itch Questionnaire scoring in the treated groups 
subgroup at the end of the study was $7.1 \pm 4$.6 points, for the levocetirizine subgroup: $7.0 \pm 4.5$ points and for the placebo subgroup: $8.6 \pm 3.4$ points, $p=0.42$ ) (Table 1 ). The BSA values were also similar in all subgroups at baseline ( $p=0.45)$, however, BSA did not reduce significantly during the study period (for details see Table 1).

The mean itch intensity before treatment was 4.6 \pm 2.8 points according to the VAS scale and $11.9 \pm 4.5$ points according to the Itch Questionnaire. Most of the patients suffered from mild (0-3 points) and moderate (3-7 points) pruritus: $36.1 \%$ and $41 \%$, respectively. Very severe itch (more than 9 points in VAS scale) was reported by $6.6 \%$ of the patients (one patient in the clemastine group and 3 patients in the levocetirizine group) $(p=0.11)$. Regarding the studied subgroups, the mean intensity of pruritus according to the VAS before treatment was $5.6 \pm 2.7$ points in the clemastine group, 4.7 \pm 3.2 points in the levocetirizine group and $3.6 \pm 1.9$ points in the placebo group $(p=0.07)$. A statistically significant decrease in mean VAS scoring was observed in clemastine and levocetirizine groups ( $p<0.001)$, but not in the placebo group. The greatest improvement of VAS was seen in the clemastine group by $2.6 \pm 3.1$ points comparing to levocetirizine (by $2.0 \pm 2.9$ points, $p=0.56$ ) and placebo (by $0.5 \pm 2.3$ points, $p=0.01$ ) groups. The difference between levocetirizine and placebo subgroups was also significant $(p=0.03)$. The relative improvement for each study subgroups is demonstrated in Figure 1.
Mean Itch Questionnaire values before treatment and on day 7 were similar in all subgroups (clemastine group: $13.3 \pm 4.7$ points, levocetirizine group: $12.2 \pm 4.6$ points, placebo group: $10.1 \pm 3.9$ points, $p=0.09$ ). Itch questionnaire scoring decreased significantly during the study in all groups. The greatest decrease was seen in the clemastine group with a change of $4.5 \pm 3.3$ points $(p<0.0001)$. The improvement of itch questionnaire scoring in the levocetirizine group was $2.2 \pm 3.4$ points and $1.9 \pm 3.5$ points in the placebo group $(p<0.01$ and 0.04 , respectively) (Figure 2 ).

The number of wrist movements during sleep did not differ significantly between groups within all analyzed nights. There was no significant difference in the number of wrist movements during sleep over the following nights when compared to the baseline night in any of the three groups (for the clemastine group $p=0.3$, for the levocetirizine group $p=0.09$, for the placebo group $p=0.84)$. In addition, no significant differences regarding the duration of the nocturnal movement activity in the studied subgroup of patients were found.

Mean DLQI values before treatment and on day $7 \mathrm{did}$ not differ significantly between groups ( $p=0.11$ and $p=$ 0.38 , respectively). A statistically significant decrease in DLQI scoring was observed on day 7 when compared to the baseline values in all groups. The greatest improvement was seen in the clemastine group $(9.7 \pm 5$. 6 points, $p<0.0001)$ followed by the levocetirizine group: $6.6 \pm 6.2$

Table 2. Scoring of pruritus and quality of life in studied patients

\begin{tabular}{|c|c|c|c|c|}
\hline Parameter & Clemastine group & Levocetirizine group & Placebo group & $P$-value \\
\hline VAS (Baseline): & & & & 0.07 \\
\hline Mean \pm SD & $5.6 \pm 2.7$ & $4.7 \pm 3.2$ & $3.3 \pm 1.8$ & \\
\hline (Range) & $(0.9-9.9)$ & $(0.8-9.4)$ & $(1.0-6.8)$ & \\
\hline VAS (EOS): & & & & 0.93 \\
\hline Mean \pm SD & $3.0 \pm 2.9$ & $2.7 \pm 3.0$ & $2.8 \pm 1.9$ & \\
\hline (Range) & $(0.1-8.9)$ & $(0-9.4)$ & $(0.4-7.0)$ & \\
\hline IQ (Baseline): & & & & 0.08 \\
\hline Mean \pm SD & $12.2 \pm 4.6$ & $13.3 \pm 4.7$ & $10.1 \pm 3.9$ & \\
\hline (Range) & $(4-22)$ & $(4-19)$ & $(4-15)$ & \\
\hline IQ (EoS): & & & & 0.48 \\
\hline Mean \pm SD & $8.5 \pm 4.5$ & $10.0 \pm 5.4$ & $8.2 \pm 4.3$ & \\
\hline (Range) & $(4-17)$ & $(3-22)$ & $(3-20)$ & \\
\hline DLQI (Baseline): & & & & 0.12 \\
\hline Mean \pm SD & $14.8 \pm 7.9$ & $14.0 \pm 6.3$ & $10.3 \pm 6.6$ & \\
\hline (Range) & $(4-30)$ & $(2-27)$ & $(3-21)$ & \\
\hline DLQI (EoS): & & & & 0.38 \\
\hline Mean \pm SD & $5.1 \pm 5.0$ & $7.4 \pm 6.0$ & $7.1 \pm 5.4$ & \\
\hline (Range) & $(1-20)$ & $(1-27)$ & $(1-17)$ & \\
\hline
\end{tabular}

EOS - end of study, IQ - Itch Questionnaire, SD - standard deviation. 
points $(p<0.0001)$ and the placebo group: $3.3 \pm 3.3$ points $(p<0.001)$ (Table 2).

\section{Discussion}

Pruritus affects approximately $70-90 \%$ of psoriatic patients. Treating itch in patients with psoriasis is challenging because its pathogenesis is still not completely understood and there are no evidence-based guidelines to make reasonable therapeutic decisions. However, several studies documented the clinical manifestation of pruritus in psoriasis. It is more common among patients with plaque-type psoriasis than among subjects with erythrodermic, guttate or pustular psoriasis $[3,18]$. The published data show that the intensity of itch in psoriasis reflected by the VAS scale is scored between 3.7 and 6.4 [3, 4, 7, 20-23]. In our study the initial mean itch intensity was within this range, i.e. $4.6 \pm 2.8$ points. Interestingly, very severe itch (more than 9 points in the VAS scale) was reported by only $6.6 \%$ of the patients while in other published studies it ranged between 18 and 33\% [3, 4, 24]. However, the methodology and sample size of these studies were different and thus the direct comparison is difficult to be made.

As mentioned above, the pathogenesis of pruritus in psoriasis is still not fully elucidated. Currently most scientists indicate that it could be related to disturbed innervation and dysregulated expression of neuropeptides in the skin [25], whereas histamine, an important itch mediator in allergic diseases, especially in urticaria, was not considered as a relevant pruritogen in psoriasis. Wiśnicka et al. [22] found no correlation between pruritus intensity and histamine plasma levels in psoriasis, as well as no difference in histamine plasma levels between pruritic and non-pruritic subjects. However, the plasma level of histamine does not necessarily have to reflect its content in the skin. The role of mast cells, which are major histamine producers in humans, is being still intensively studied. Harvima et al. [26] found that psoriasis is characterized by an increased number of mast cells in the upper dermis and in epidermis while Schubert and Christophers [27] observed that degranulated mast cells are seen at the very early stages of psoriatic inflammation in the skin. Using microdialysis technique Petersen et al. [28] found that the histamine level was increased in the involved psoriasis skin compared to the normal skin in the controls and uninvolved psoriatic skin. They treated 16 patients with high-dose ranitidine for 6 months observing PASI reduction and a decrease in histamine concentration in psoriatic skin lesions without any significant reduction in mast cell number. Furthermore, Gschwandtner et al. [29] found that the most recently described histamine $\mathrm{H}(4)$ receptor is highly expressed on plasmacytoid dendritic cells (pDC) in psoriasis and that histamine influences cytokine production and migration of pDC. In addition, Mommert et al. [30] showed that stimulation by histamine or $\mathrm{H} 4$ receptor agonist increases production of IL-17 by Th17 cells, which is thought to play a crucial role in the pathogenesis of psoriasis. These observations suggested that skin mast cells in active psoriasis might be functionally hyperreactive and that histamine may be involved in the pathogenesis of psoriasis.

Based on our own observations, antihistamines are commonly used to treat itch in psoriatic patients in clinical practice, mainly the first-generation ones due to their additional sedative effect, despite they have never been shown effective in any controlled study. Other authors also mentioned antihistamine prescriptions. Prignano et al. [24] pointed new antihistamines as being effective in only $13 \%$ of psoriatic patients with pruritus and only for a short time. Similarly, Amatya et al. [3] found that some psoriatics used antihistamines but all complained about very short effectiveness of these drugs. In turn, Yosipowitch et al. [4] showed that antihistamines as antipruritic treatment were used by $45 \%$ of patients, among whom hydroxyzine was the most often used drug. An antipruritic effect was short in the majority of patients (84\%), while $16 \%$ reported lack of any itch reduction.

In the light of the above-mentioned studies, we have found our results quite surprising. At the beginning, we expected that antihistamines would not improve itch in plaque-type psoriasis and any potential effect would concern only sedative drugs of first-generation antihistamines. However, we have found a statistically significant decrease in mean VAS scoring not only in the clemastine group but also in the levocetirizine group. A significant improvement was seen in the clemastine group on the second day and in the levocetirizine group on the third day. Patients in the placebo group did not achieve any significant improvement of itch during the whole treatment period of 7 days $(p=0.48)$. Itch intensity according to the Itch Questionnaire decreased significantly in all treatment groups, but again the greatest improvement was seen in the clemastine group followed by the levocetirizine group and the placebo group. The effect in the placebo group might be attributed to the antipsoriatic treatment given to all patients, however, a significantly larger itch reduction in the other groups might indicate the possible additional effect of histamine blockade.

Lack of change of wrist movements during the entire period of study requires further investigation, but in our opinion, this method is still not well validated for itch intensity measurements and a number of co-factors might influence the final results achieved with such instruments. Similar observations come from other studies which used actigraphy as a possibly objective method of scratching behavior assessment. Murray and Rees [31], in their study including 117 patients with atopic dermatitis, psoriasis, cholestasis and idiopathic itch, found no correlation between VAS scoring and actigraphy measurements. In the study of Bender et al. [32], scratching measured by accelerometers did not correlate with results 
of subjective methods of evaluating pruritus. Bringhurst et al. [18] made similar observations - there was no relationship between VAS scoring in adults with pruritus and actigraphy results. In contrast, they found a statistically significant correlation in the pediatric population $(r=0.4$, $p=0.049$ ). Bringhurst et al. [18] suggested that the difference between adults and children could be due to the fact that among adults (especially in elderly) insomnia and concomitant diseases are more common and they can influence their night activity regardless of pruritus. However, in another large randomized study with 336 children with eczema (aged 6 months to 16 years) conducted by Wootton et al. [33], actigraphy did not correlate well with disease severity or quality of life when used as an objective outcome measure and was not responsive to change over time. They noticed, just like we did in our study, the problem in distinguishing between eczemarelated and eczema-nonrelated movements (so-called restless movements) which could be related to other factors like nightmares, anxiety, concomitant diseases or high temperature in the room at night. Although actigraphy seems to be a very promising method of objective itch assessing, further work is needed to establish improved ways of analyzing obtained data. The first steps were made by Feuerstein et al. [34] who used k-means cluster analysis to differentiate scratching from walking and restless sleep, which are potential confounds for nighttime scratching. This work indicated that the features described there can be used to develop a classifier that discriminates scratch from other activities. Whether indeed such algorithms would be of help will be shown in the future.

\section{Conclusions}

Pruritus in psoriasis is a frequent and burdensome symptom of the disease and its treatment remains challenging. Studies on itch pathogenesis as well as randomized, placebo-controlled studies on new and old drugs with a potential antipruritic effect are highly needed.

\section{Conflict of interest}

The authors declare no conflict of interest

\section{References}

1. Langley RG, Krueger GG, Griffiths CE. Psoriasis: epidemiology, clinical features, and quality of life. Ann Rheum Dis 2005; 64: 18-23.

2. Reich A, Hrehorów E, Szepietowski JC. Pruritus is an important factor negatively influencing the well-being of psoriatic patients. Acta Derm Venereol 2010; 90: 257-63.

3. Amatya B, Wennersten G, Nordlind K. Patients' perspective of pruritus in chronic plaque psoriasis: a questionnairebased study. J Eur Acad Dermatol Venereol 2008; 22: 822-6.
4. Yosipowitch G, Goon A, Wee J, et al. The prevalence and clinical characteristics of pruritus among patients with extensive psoriasis. Br J Dermatol 2000; 143: 969-73.

5. Szepietowski JC, Reich A, Wiśnicka B. Itching in patients suffering from psoriasis. Acta Dermatovenerol Croat 2002; 10: 221-6.

6. Sampogna F, Gisondi P, Melchi CF, et al. Multipurpose Psoriasis Research on Vital Experiences Investigators. Prevalence of symptoms experienced by patients with different clinical types of psoriasis. Br J Dermatol 2004; 151: 594-9.

7. Reich A, Szepietowski JC, Wiśnicka B, Pacan P. Does stress influence itching in psoriatic patients? Dermatol Psychosom 2003; 4: 151-5.

8. Gupta MA, Gupta AK, Kirby S, et al. Pruritus in psoriasis. A prospective study of some psychiatric and dermatologic correlates. Arch Dermatol 1988; 124: 1052-7.

9. Szepietowski J, Adamski Z, Chodorowska G, et al. Guidelines of Polish Dermatological Society on the treatment of psoriasis vulgaris and arthropathic psoriasis (psoriatic arthritis) with biological drugs. Przegl Dermatol 2010; 97: 1-13.

10. Szepietowski J, Adamski Z, Chodorowska G, et al. Diagnostics and treatment of psoriasis vulgaris: guidelines of the Polish Dermatological Society. Part I: mild psoriasis, psoriasis in children. Przegl Dermatol 2012; 99: 83-96.

11. Fredriksson T, Pettersson U Severe psoriasis - oral therapy with a new retionoid. Dermatologica 1978; 157: 238-44.

12. Puzenat E, Bronsard V, Prey S, et al. What are the best outcome measures for assessing plaque psoriasis severity? A systematic review of the literature. J Eur Acad Dermatol Venereol 2010; 24: 10-6.

13. Matterne U, Strassner T, Apfelbacher CJ, et al. Measuring the pravalence of chronic itch in the general population: development and validation of a questionnaire for use in largescale studies. Acta Derm Venereol 2009; 89: 250-6.

14. Szepietowski J, Salomon J, Finlay AY, et al. Dermatology Life Quality Index: Polish version. Derm Klin 2004; 6: 63-70.

15. Phan NQ, Blome C, Fritz F, et al. Assessment of pruritus intensity: prospective study on validity and reliability of the visual analogue scale, numerical rating scale and verbal rating scale in 471 patients with chronic pruritus. Acta Derm Venereol 2012; 92: 502-7.

16. Szepietowski J, Reich A. Świąd. Patomechanizm, klinika, leczenie. Termedia, Poznań 2010.

17. Muszer K, Reich A. Creation and validation of the questionnaire for assessment of itch severity. Abstract book. II International Students' Conference of Young Medical Researchers, Wrocław, 13-14.04.2012, 203.

18. Bringhurst C, Waterston K, Schofield O, et al. Measurement of itch using actigraphy in pediatric and adult populations. J Am Acad Dermatol 2004; 51: 893-8.

19. Yngman-Uhlin P, Johansson A, Fernström A, et al. Fragmented sleep: an unrevealed problem in peritoneal dialysis patients. Scand J Urol Nephrol 2011; 45: 206-15.

20. Reich A, Heisig M, Phan NQ, et al. Visual analogue scale: evaluation of the instrument for the assessment of pruritus. Acta Derm Venereol 2012; 92: 497-501.

21. Remröd C, Lonne-Rahm S, Nordlind K. Study of substance $\mathrm{P}$ and its receptor neurokinin-1 in psoriasis and their relation to chronic stress and pruritus. Arch Dermatol Res 2007; 299: 85-91.

22. Wiśnicka B, Szepietowski JC, Reich A, et al. Histamine, substance $P$ and calcitonin-gene related peptide plasma concentration and pruritus in patients suffering from psoriasis. Dermatol Psychosom 2004; 5: 73-8. 
23. Verhoeven EW, Kraaimat FW, Jong EM, et al. Effect of daily stressors on psoriasis: a prospective study. I Invest Dermatol 2009; 129: 2075-7.

24. Prignano F, Ricceri F, Pescitelli L, et al. Itch in psoriasis: epidemiology, clinical aspects and treatment options. Clin Cosmet Investig Dermatol 2009; 2: 9-13.

25. Reich A, Szepietowski J. Mediators of pruritus in psoriasis. Mediators Inflamm 2007; 2007: 64727.

26. Harvima IT, Nilsson G, Suttle MM, et al. Is there a role for mast cells in psoriasis? Arch Dermatol Res 2008; 300: 461-78.

27. Schubert C, Christophers E. Mast cells and macrophages in early relapsing psoriasis. Arch Dermatol Res 1985; 277 : 352-8.

28. Petersen LJ, Hansen U, Krisensen JK, et al. Studies on mast cells and histamine release in psoriasis: effect of ranitidine. Acta Derm Venereol 1998; 78: 190-3.

29. Gschwandtner M, Mommert S, Köther B. The histamine H4 receptor is highly expressed on plasmacytoid dendritic cells in psoriasis and histamine regulates their cytokine production and migration. J Invest Dermatol 2011; 131: 1668-76.

30. Mommert S, Gschwandtner M, Koether B. Human memory Th17 cells express a functional histamine $\mathrm{H} 4$ receptor. Am J Pathol 2012; 180: 177-85.

31. Murray CS, Rees JL. Are subjective accounts of itch to be relied on? The lack of relation between visual analogue itch scores and actigraphic measures of scratch. Acta Derm Venereol 2011; 91: 18-23.

32. Bender BG, Ballard R, Canono B, et al. Disease severity, scratching, and sleep quality in patients with atopic dermatitis. J Am Acad Dermatol 2008; 58: 415-20.

33. Wootton Cl, Koller K, Lawton S, et al. Are accelerometers a useful tool for measuring disease activity in children with eczema? Validity, responsiveness to change, and acceptability of use in a clinical trial setting. Br J Dermatol 2012; 167: 1131-7.

34. Feuerstein J, Austin D, Sack R, et al. Wrist actigraphy for scratch detection in the presence of confounding activities. Conf Proc IEEE Eng Med Biol Soc 2011; 2011: 3652-5. 